\title{
Quetiapine use Resulting in Severe Rhabdomyolysis: A Rare Adverse Effect
}

\author{
Ansari J*, Anwar S \\ Department of Internal Medicine, Wright State \\ University, USA \\ *Corresponding author: Ansari J , Department of \\ Internal Medicine, Wright State University, Boonshoft \\ School of Medicine, 1 Wyoming St, Dayton, Oh 45409, \\ USA
}

Received: December 31, 2016; Accepted: February 16, 2017; Published: February 18, 2017

\section{Abstract}

Quetiapine is one of the common second-generation antipsychotic drugs that are used to treat behavioral disorders. Only few cases of Quetiapineinduced rhabdomyolysis have been described in the literature. We report a case of severe rhabdomyolysis, which resulted from quetiapine use in a patient who was on it for the treatment of bipolar disorder for about 8 months. Our case highlights the importance of monitoring for these rare adverse effects.

Keywords: Quetiapine; Rhabdomyolysis; Creatine phosphokinase

\section{Introduction}

In current psychiatric medicine, Quetiapine is one of the common atypical antipsychotic drugs, which are used due to its therapeutic efficiency and clinical safety [1]. Its indication includes treatment of schizophrenia, moderate to severe manic episodes and major depressive episodes in bipolar disorder [1,2]. There are studies, which also indicate its use in patients with delirium [3]. Its frequent use is due to its low propensity to induce extrapyramidal side effects. We report a case of rhabdomyolysis, which resulted from quetiapine use. This rare adverse effect has only been noted in very few cases.

\section{Case Presentation}

A 56-year-old male was admitted to the hospital for left arm pain and right lower extremity pain for 1 day. He had a known medical history of bipolar disorder, hypertension, and cardiomyopathy. His medications include Quetiapine $200 \mathrm{mg} /$ day, Restoril $15 \mathrm{mg} /$ day, Metoprolol $25 \mathrm{mg}$ /day and Lisinopril $2.5 \mathrm{mg} /$ day. Patient was taking quetiapine for 8 months patient denied any kind of recent trauma or extraneous activity. No falls or chest pain. His vital signs on admission were within normal limit. Exam showed tender right lower extremity, otherwise rest of the exam was normal. The laboratory findings on admission revealed Creatine Phosphokinase (CPK) level of 14.902 U/L, myoglobin 1278 ng/ml, Aspartate Aminotransferase (AST) 769 U/L, Alanine Aminotransferase ( ALT) 967 U/L, Troponin $0.41 \mathrm{ng} /$ $\mathrm{ml}$ and Creatinine $1.8 \mathrm{mg} / \mathrm{dl}$. Patient's baseline renal function was around $0.9 \mathrm{mg} / \mathrm{dl}$. Urine drug screen was positive for benzodiazepine, whichresulted due to the fact that patient received lorazepam in the emergency department and urine drug screen was obtained later. Right upper quadrant ultrasound was unremarkable. Patient was started on intravenous fluids; his renal function was monitored along with urinary output. Quetiapine was discontinued but Restoril was continued. Patient's CPK level continued to improve and renal function normalized. Troponins were also trended and continued to decline. Liver function enzymes (LFTs) normalized as well. Therefore, this patient was considered to have rhabdomyolysis due to quetiapine treatment. Patient was discharged to home after being in the hospital for few days $[5,6]$.

\section{Discussion}

Quetiapine is an atypical antipsychotic of the dibenzothiazepine class, which is administered orally and is indicated for behavioral disorders such as schizophrenia and bipolar disorder [2,4]. It is also used as a sleep aid sometimes due to its sedating effect but that is not recommended. Liver through cytochrome P450 isoenzyme metabolizes it $[2,7]$. It is available in strengths ranging from 25-400 $\mathrm{mg}$ [2]. Some of the common adverse effects with its use include somnolence, orthostatic hypertension, high blood pressure and elevated triglycerides $[2,8,9]$. But in rare cases prolonged QT interval, cardiac arrhythmias and neuroleptic malignant syndrome have been seen $[8,9]$. Thus far only handful cases of severe rhabdomyolysis following quetiapine overdose or therapeutic dosage have been described in the literature. Our case is different and to our knowledge, this is the first case where patient developed rhabdomyolysis after being treated with quetiapine for 8 months. There was one case report which we came across while reviewing the literature where patient developed rhabdomyolysis after 6-month use of quetiapine but that patient also had chronic kidney disease [10]. It is also worth stating that the Swiss pharmacological guide did mention a risk of diffuse muscular pain in association with therapeutic dosage of quetiapine [11]; but our case is different, as our patient was tolerating the medication well before this event. Our patient was not on any other medication, which would contribute to rhabdomyolysis. We can also dismiss the possibility of Neuroleptic malignant syndrome due to the absence of neurological signs, hyperthermia and autonomic dysfunction.

Considering the frequent use of quetiapine in behavioral disorders, we would emphasize that clinicians should be vigilant of this rare adverse effect in a patients who presents with severe rhabdomyolysis and is on quetiapine.

\section{References}

1. Velasco-Montes J, Oriñuela-González I, Sanjuán-López AZ. Rhabdomyolysis secondary to quetiapine. Actas Esp Psiquiatr. 2012; 40: 97-99.

2. Gibiino S, Trappoli A, Balzarro B, Atti AR, De Ronchi D. Coma after Quetiapine Fumarate Intentional overdose in a 71-year-old Man: A Case Report. Drug Safety - Case Reports. 2015; 2: 3.

3. Maneeton B, Maneeton N, Srisurapanont M, Chittawatanarat K. Quetiapine 
versus haloperidol in the treatment of delirium: a double-blind, randomized, controlled trial. Drug Design, Development and Therapy. 2013; 7: 657-667.

4. Hasselstrom J, Linnet K. Quetiapine serum concentrations in psychiatric patients: the influence of comedication. Ther Drug Monit. 2004; 26: 486-491.

5. Jahn $\mathrm{H}$, Kiefer F, Arlt J. Rhabdomyolysis with quetiapine.

6. Dickmann JR, Dickmann LM. An uncommonly recognized cause of rhabdomyolysis after quetiapine intoxication. American Journal of Emergency Medicine. 2010; 28: 1060.e1-1060.e2.

7. DeVane $\mathrm{CL}$, Nemeroff $\mathrm{CB}$. Clinical pharmacokinetics of quetiapine: an atypical antipsychotic. Clin Pharmacokinet. 2001; 40: 509-522.
8. Taylor D, Paton C, Kapur S. The Maudsley prescribing guidelines in psychiatry. West Sussex: Wiley-Blackwell. 2012

9. Jump up to: a b c "PRODUCT INFORMATION STADA(TM) Quetiapine (quetiapinefumarate Tablets $25 \mathrm{mg}, 100 \mathrm{mg}, 200 \mathrm{mg}, 300 \mathrm{mg}$ )". TGA eBusiness Services. STADA Pharmaceuticals Australia Pty Limited. 30 November 2012

10. Ceri M, Unverdi S, Altay M, Duranay M. Comment on: low-dose quetiapineinduced severe Rhabdomyolysis. Renal Failure. 2011; 33: 463-464.

11. Compendium Suisse des medicaments. 2012.
Austin J Clin Case Rep - Volume 4 Issue 1 - 2017

ISSN : 2381-912X | www.austinpublishinggroup.com

Ansari et al. (C) All rights are reserved
Citation: Ansari J, Anwar S. Quetiapine use Resulting in Severe Rhabdomyolysis: A Rare Adverse Effect. Austin J Clin Case Rep. 2017; 4(1): 1113. 